\title{
Design and Simulation of Continuously Tunable Multiband Terahertz Metamaterials
}

\author{
Xiaoqing Zhao, Bin Yang*, Jingquan Liu, \\ Gang Tang ${ }^{1}$ and Chunsheng Yang \\ National Key Laboratory of Science and Technology on Micro/Nano Fabrication, \\ Department of Micro/Nano Electronics, Shanghai Jiao Tong University, \\ 800 Dongchuan Road, Minhang District, Shanghai 200240, China \\ 'Department of Mechanical Electrical Engineering, Nanchang Institute of Technology, \\ 289 Tianxiang Avenue, Changdong Town, Nanchang County, Nanchang City, \\ Jiangxi Province 330099, China
}

(Received July 17, 2015; accepted August 28, 2015)

Key words: MEMS, metamaterials, terahertz, multiband, continuous tunability

In this work, two types of metamaterial based on four symmetrical arc-shaped and line-shaped " $T$ " cantilevers are investigated as resonator arrays, which can be structurally reconfigured by a thermal actuation mechanism. The simulation results show that both of the proposed metamaterial structures provide not only a resonant multiband-frequency operation but also polarization-independent tunability. The three resonance frequencies can be continuously adjusted in the range from 0.5000 to $2.5000 \mathrm{THz}$ when the angles between the released cantilevers and the silicon substrate are changed at different temperatures. By investigating the electric and magnetic field strength distributions at the resonant frequencies, we have concluded that the multiband absorptions are caused by dipole-type resonance mechanisms. Our proposed tunable metamaterials have some advantages, namely, simple resonant structure, mechanism, and multiband operation frequencies in a narrow frequency range; these are highly desirable for $\mathrm{THz}$ applications in high-sensitivity sensing, tunable filtering, switching, frequency-selective detection, and multispectral imaging.

\section{Introduction}

The terahertz $(\mathrm{THz})$ region has attracted much research interest for the realization of promising and conventionally unattainable applications owing to the several unique properties of $\mathrm{THz}$ waves; applications include invisibility cloaking devices, ${ }^{(1)}$ highresolution superlenses, ${ }^{(2)}$ perfect absorbers, ${ }^{(3)}$ sensors, ${ }^{(4)}$ filters, ${ }^{(5)}$ nondestructive testing, ${ }^{(6)}$ and toroid responses. ${ }^{(7)}$ However, because naturally existing materials fall short of an

${ }^{*}$ Corresponding author: e-mail: binyang@sjtu.edu.cn 
appropriate response at some available frequencies, there are critical limitations to many practical applications in the THz field. Therefore, artificially structured electromagnetic materials have been widely studied for creating novel electromagnetic properties that are not available in natural materials, such as negative refractive index, ${ }^{(8)}$ perfect focusing, ${ }^{(9)}$ cloaking, ${ }^{(10)}$ polarization manipulation, ${ }^{(11)}$ and phase discontinuities. ${ }^{(12)}$ These extreme electromagnetic properties are obtained through the interaction of incident electromagnetic waves with metamaterial structures, which are designed with different shapes and sizes. As a result, the interaction of the incident electromagnetic waves can be actively controlled as the metamaterial pattern shape is changed through external force actuation. These actuation mechanisms include optical, ${ }^{(13)}$ mechanical, ${ }^{(14)}$ electrical, ${ }^{(15)}$ magnetic, ${ }^{(16)}$ and thermal ${ }^{(17)}$ methods.

Recently, the tunability of metamaterials has received increasing attention, because the active control of metamaterial characteristics would enable their properties to be engineered in a desired way. It was suggested that the approach to metamaterial tunability has been demonstrated, for example, by semiconductor diodes ${ }^{(18)}$ to vary the capacitance of resonator media or photoexcitation, ${ }^{(19)}$ external voltages, ${ }^{(20)}$ electrostatic actuation, ${ }^{(21)}$ external magnetostatic fields, ${ }^{(22)}$ or thermal bimorphs ${ }^{(23)}$ to change the property of surrounding media, ${ }^{(19)}$ and flexible substrates to achieve continuous deformation. (24) Structural tunability is an efficient way of controlling amplitude, polarization, or directionality for metamaterial structures so that metamaterial properties can be directly modified by reconfiguring the unit cell. Metamaterials with multipleresonance excitations have provided access to unique and potential devices.

In this study, two structural multiband metamaterials with bimorph cantilevers were designed and simulated: micro-electromechanical system (MEMS)-based four symmetrical arc-shaped and line-shaped "T" cantilevers, respectively. The proposed MEMS-based metamaterial structures are geometrically reconfigurable and can be easily scaled by thermally reconfiguring the metamaterial unit cells. Furthermore, the upwardbending bimorph cantilevers can result in out-of-plane tunable deformation without the need for complicated fabrication processes. Using the thermal actuation mechanism, the bending degree of the MEMS-based bimorph cantilevers can be dynamically adjusted to achieve a tunable electromagnetic response in metamaterials.

\section{Structural Design and Simulation}

\subsection{Structural design}

In this study, two new types of metamaterial structure are proposed by changing the edge shape of the " $\mathrm{T}$ " cantilevers, which are composed of parylene-C bottom and gold top layers. These two modified metamaterial structures on a silicon substrate are expected to exhibit tunable multiband transmission characteristics. To simplify the expression, the arc-shaped and line-shaped " $\mathrm{T}$ " cantilever split ring resonator (SRR) structures are referred as T-SRR01 and T-SRR02, which are shown schematically in Figs. 1(a) and 1(b), respectively. The detailed dimensions of T-SRR01 and T-RR02 are shown in Table 1. The adjustable angle between the released cantilever tip and the substrate is expressed by "O". The capacitance of the T-SRR structures depends on the deformation 


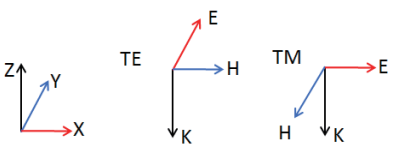

Fig. 1. (Color online) Schematic of the released unit cells for (a) T-SRR01 and (b) T-SRR02 arrays.

Table 1

Structural parameters of the T-SRRs.

\begin{tabular}{lccccccc}
\hline Device & $\begin{array}{c}\text { Length } L \\
(\mu \mathrm{m})\end{array}$ & $\begin{array}{c}\text { Beam } \\
\text { width } W \\
(\mu \mathrm{m})\end{array}$ & $\begin{array}{c}\text { Split wall } \\
\text { width } I \\
(\mu \mathrm{m})\end{array}$ & $\begin{array}{c}\text { Split gap } \\
\text { width } G \\
(\mu \mathrm{m})\end{array}$ & $\begin{array}{c}\text { Period } P \\
(\mu \mathrm{m})\end{array}$ & $\begin{array}{c}\text { Parylene-C } \\
\text { thickness } \\
(\mu \mathrm{m})\end{array}$ & $\begin{array}{c}\text { Gold } \\
\text { thickness } \\
(\mu \mathrm{m})\end{array}$ \\
\hline T-SRR01 & 40 & 4 & - & 4 & 130 & 2 & 0.5 \\
T-SRR02 & 40 & 4 & 72 & - & 130 & 2 & 0.5 \\
\hline
\end{tabular}

angle of the cantilevers. Currently, the most reported MEMS-based metamaterials are actuated by electrical methods. However, for our designed structures, the anchor of the cantilever is located in the center. Hence, the thermal actuation mechanism is selected to adjust the deformation angle. Figures $1(\mathrm{a})$ and (b) show the released unit cells of the T-SRRs, which are bent upward due to the tensile stress of the cantilever. The incident electric fields for transverse electric (TE) and transverse magnetic (TM) polarizations are perpendicular and parallel to the $x$-axis, respectively, as shown in Fig. 1.

\subsection{Electromagnetic simulation}

Finite-difference time-domain (FDTD) numerical simulations were conducted to calculate the transmission spectra and the electromagnetic field distributions corresponding to the resonance modes with normally incident $\mathrm{THz}$ waves of either TE or TM polarization using Computer Simulation Technology (CST) software. Full-field electromagnetic wave simulations were performed with built-in material parameters ( $\mathrm{Au}, \mathrm{Si}$, and user-defined parylene-C) from the software's material data. Parylene $\mathrm{C}$ film is a transparent polymer film, which has excellent electrical and chemical inertness, conformal coating, barrier, and physical properties, and good biocompatibility. It has been used in biomedical devices and microelectronics. Therefore, parylene-C was selected as the insulating dielectric layer in this work. The material parameters of $\mathrm{Au}$, $\mathrm{Si}$, and user-defined parylene-C are listed in Table 2. In the simulation, a single unit cell of the metamaterial was simulated under periodic boundary conditions employed in axial directions orthogonal to the incident waves. The perfectly matched layers are applied in 
Table 2

Material properties of simulated structures.

\begin{tabular}{lccccc}
\hline Materials & Permittivity & Permeability & $\begin{array}{c}\text { Young's } \\
\text { modulus } \\
(\mathrm{GPa})\end{array}$ & $\begin{array}{c}\text { Poisson's } \\
\text { ratio }\end{array}$ & $\begin{array}{c}\text { Coefficient of } \\
\text { thermal expansion } \\
\left(\times 10^{-6} \mathrm{~K}^{-1}\right)\end{array}$ \\
\hline $\mathrm{Si}$ & 11.9 & 1 & 169 & 0.3 & 2.568 \\
Parylene-C & 2.9 & 1 & 4.5 & 0.4 & 12.8 \\
$\mathrm{Au}$ & - & 1 & 78 & 0.4 & 14.2 \\
\hline
\end{tabular}

the direction of propagation of the electromagnetic waves. Plane waves were incident on the unit cell, and the transmission spectrum was monitored with a power monitor placed above the structure. Meanwhile, frequency profile monitors were used to detect the electric and magnetic fields.

\section{Results and Discussion}

Because the T-SRR structure is symmetrical, the transmission is the same for both TE and TM polarizations. The simulated transmission spectra of T-SRR01 and T-SRR02 are shown in Figs. 2(a) and 2(b), respectively. The three resonant frequencies of T-SRR01 and T-SRR02 are referred as T-SRR01-1, T-SRR01-2, T-SRR01-3, and T-SRR02-1, T-SRR02-2, T-SRR02-3, respectively. The T-SRR01-2 has very sharp peaks referred as Fano resonance, which has a fixed frequency of $1.8000 \mathrm{THz}$. O angles are varied from 10 to 2 degrees. (The relative spectral width $\Delta f / f_{\text {res }}$ with $\Delta f$, the spectral width at half maximum of the absorbance spectra, are presented in Table 3). The resonant frequency ranges for T-SRR01 and T-SRR02 resonator arrays have a redshift when the deformation angle decreases from 10 to 0 degrees. As we can see, the resonant frequencies of T-SRR02 arrays have a slight redshift compared with T-SRR01 resonator arrays, which may result from the different edge shapes of the " $\mathrm{T}$ " cantilevers.

To investigate the tunability of the released T-SRR01 and T-SRR02, the dependence of the resonance frequency on the angle $O$ between the silicon and the cantilever is plotted in Fig. 3. As shown in Fig. 3, for the first resonant frequency of T-SRR01 and T-SRR02, the frequency of T-SRR01-1 decreases from 0.78667 to $0.61006 \mathrm{THz}$, while the frequency decreases from 0.71765 to $0.5451 \mathrm{THz}$ for T-SRR02-1 when the angle O decreases from 10 to 0 degrees. For the second resonance, the frequencies of T-SRR01-2 and T-SRR02-2 decrease from 1.7956 to $1.6778 \mathrm{THz}$ and from 1.7814 to $1.4931 \mathrm{THz}$, respectively, as the angle $\mathrm{O}$ is also decreased from 10 to 0 degrees. For the third resonance, the frequencies of T-SRR01-3 and T-SRR02-3 decrease from 2.0981 to 1.6778 $\mathrm{THz}$ and from 1.8709 to $1.8605 \mathrm{THz}$, respectively, as the angle $\mathrm{O}$ is decreased from 10 to 2 and 10 to 0 degrees, respectively. By comparing the tunable range of the resonance frequencies of these two resonators, it is found that T-SRR02-2 has a larger tunable range of $0.2883 \mathrm{THz}$ for the second resonance, while T-SRR01-3 has the larger tunable range of $0.2981 \mathrm{THz}$ for the third resonance. 


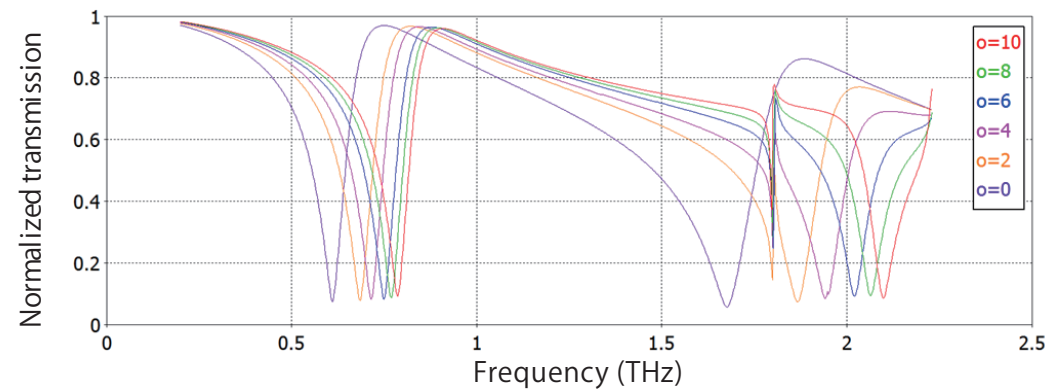

(a)

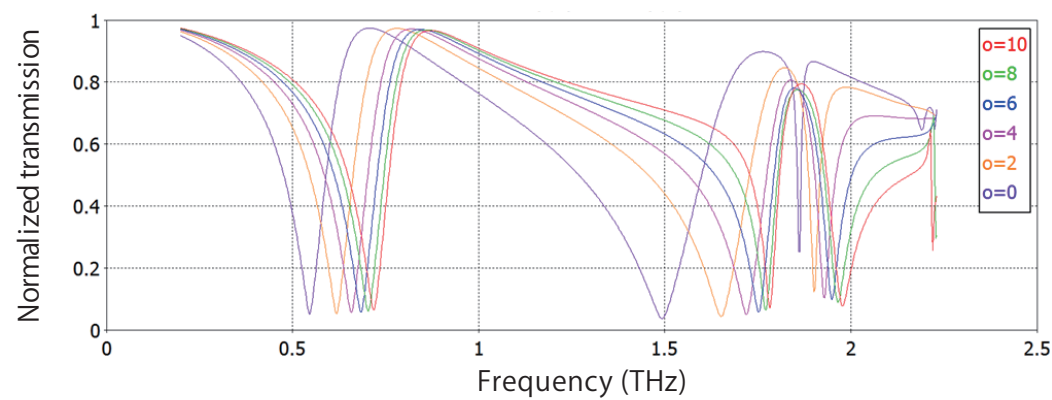

(b)

Fig. 2. (Color online) Simulated transmission spectra of (a) T-SRR01 and (b) T-SRR02 arrays with an incident $\mathrm{THz}$ wave.

Table 3

Relative spectral width of T-SRR01-2 absorbance spectra.

\begin{tabular}{lccccc}
\hline Angle O & $2^{\circ}$ & $4^{\circ}$ & $6^{\circ}$ & $8^{\circ}$ & $10^{\circ}$ \\
\hline TE/TM & 0.012 & 0.009 & 0.006 & 0.005 & 0.005
\end{tabular}

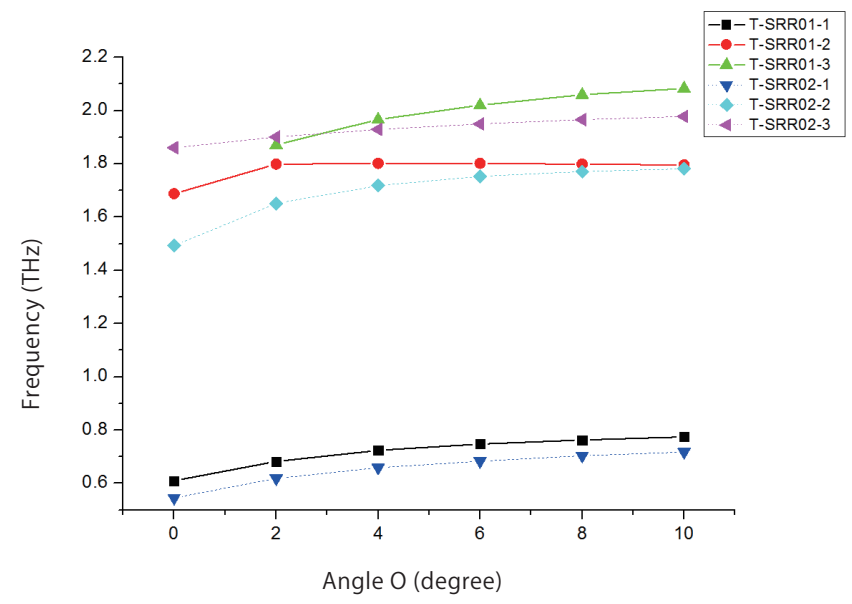

Fig. 3. (Color online) Dependence of resonant frequency on angle $\mathrm{O}$ between the silicon and the cantilever. The solid and dotted lines represent the resonance frequencies for T-SRR01 and T-SRR02, respectively. 
To further investigate this resonance mode, FDTD simulations were performed to calculate the electric and magnetic field strength distributions at the resonance frequencies. Electric and magnetic field strength distributions corresponding to the resonance frequencies in Figs. 2(a) and 2(b) are shown in Figs. 4(a)-4(e) and 5(a)-5(f),

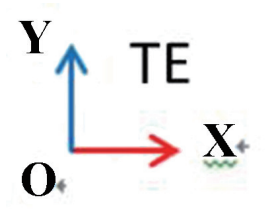

(a)
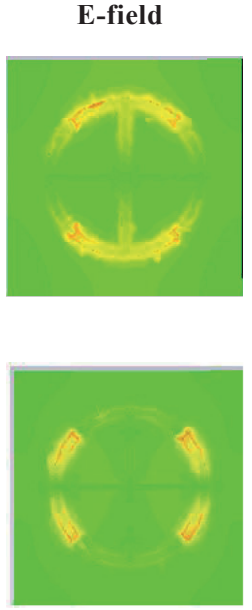

(b)

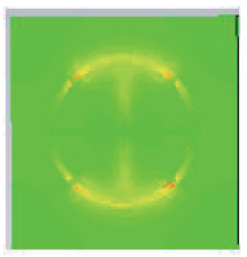

(d)

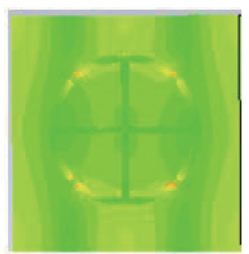

(c)

$(\mathrm{d})$

(e)

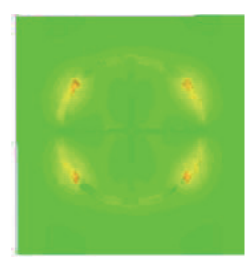

H-field
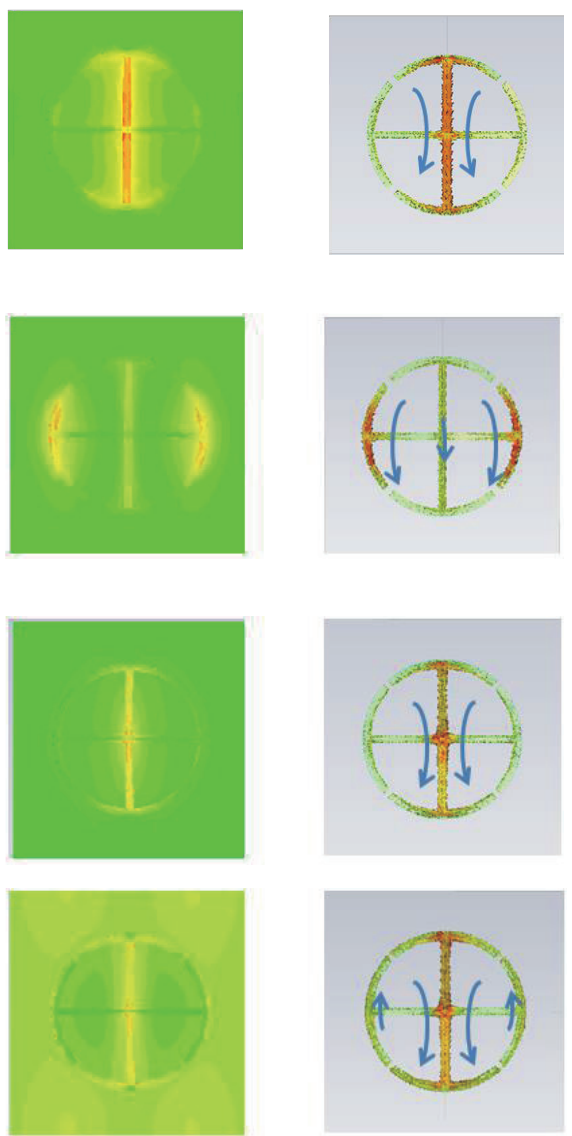

Surface current
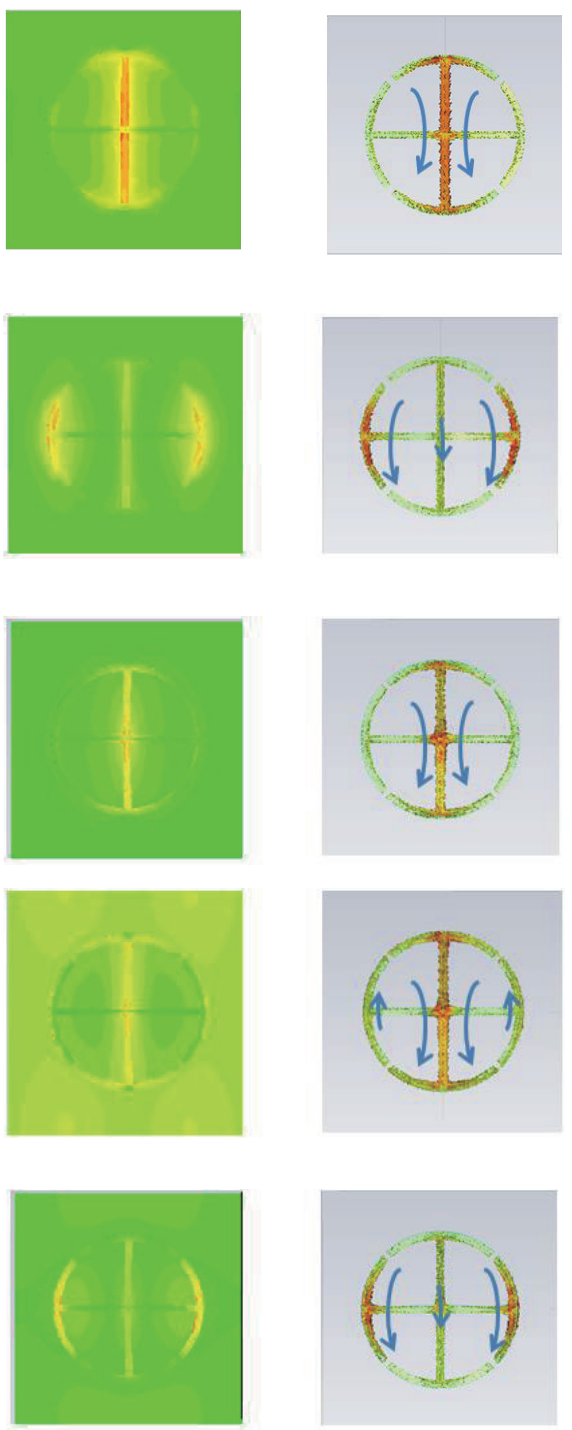

Fig. 4. (Color online) The calculated electric and magnetic field distributions and the surface current of the resonances in Fig. 2(a) are shown for (a) T-SRR01-1 (0.61006 THz), (b) T-SRR01-2 $\left(\mathrm{O}=0^{\circ}\right)(1.6778 \mathrm{THz}),(\mathrm{c}) \mathrm{T}-\mathrm{SRR} 01-1(0.78667 \mathrm{THz}),(\mathrm{d}) \mathrm{T}-\mathrm{SRR} 01-2(1.7956 \mathrm{THz})$, and $(\mathrm{e})$ T-SRR01-3 $\left(\mathrm{O}=10^{\circ}\right)(2.0981 \mathrm{THz})$. 
(a)
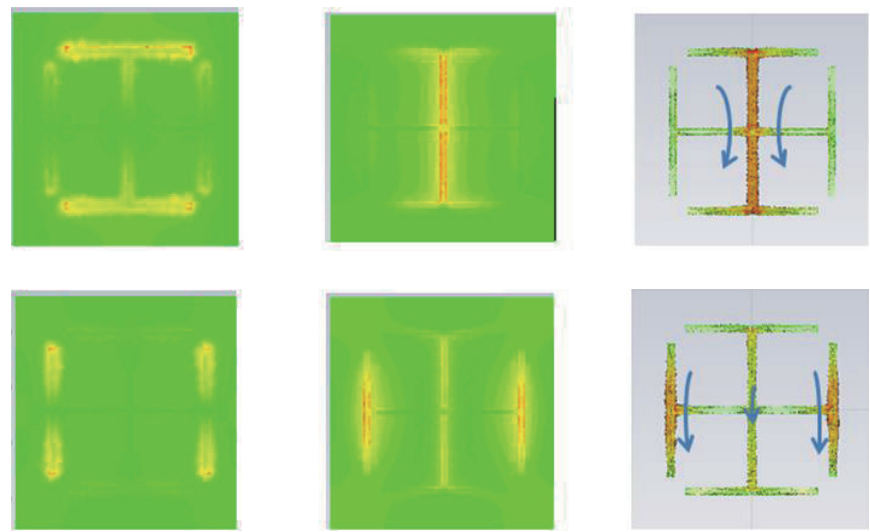

(b)
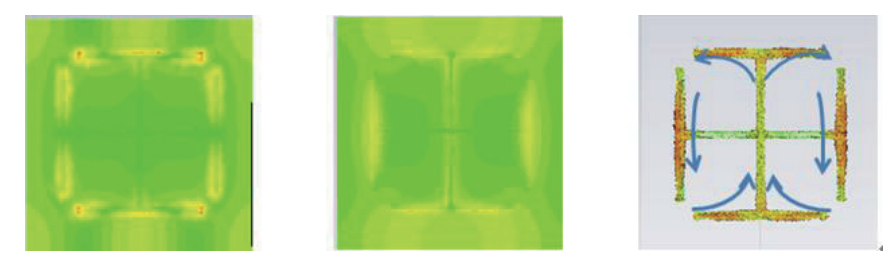

(d)
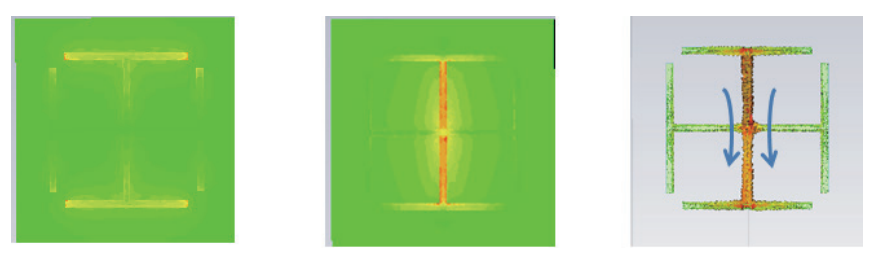

(e)
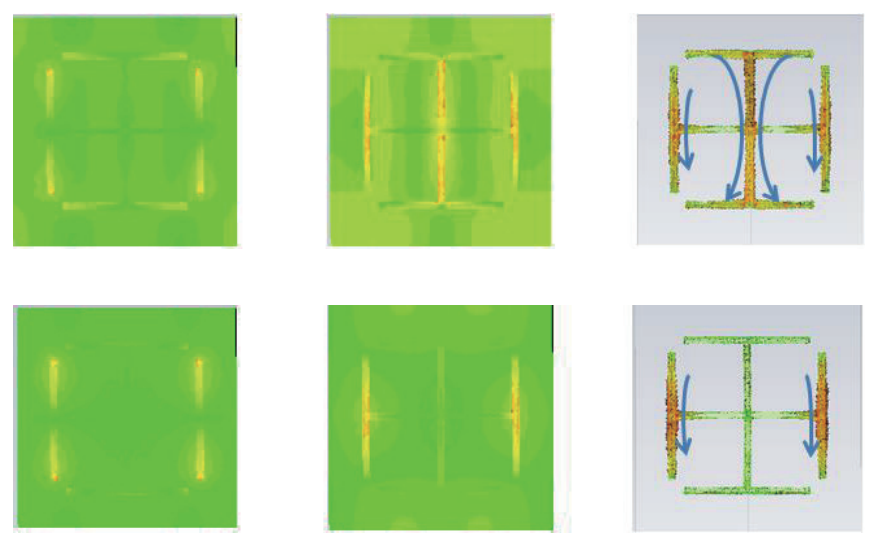

Fig. 5. (Color online) The calculated electric and magnetic field distributions and the surface current of the resonances in Fig. 2(b) are shown for (a) T-SRR02-1 (0.5451 THz), (b) T-SRR02-2 $(1.4931 \mathrm{THz}),(\mathrm{c}) \mathrm{T}-\mathrm{SRR} 02-3\left(\mathrm{O}=0^{\circ}\right)(1.8605 \mathrm{THz}),(\mathrm{d}) \mathrm{T}-\mathrm{SRR} 02-1(0.71765 \mathrm{THz}),(\mathrm{e})$ T-SRR02-2 $(1.7814 \mathrm{THz})$, and (f) T-SRR02-3 $\left(\mathrm{O}=10^{\circ}\right)(1.9783 \mathrm{THz})$. 
respectively. For the first resonant frequency of T-SRR01, the charges accumulate strongly in the edge of the arc-shaped " $T$ " cantilevers inducing harmonic current along the $y$-axis. The magnetic field strength is primarily distributed along the cantilevers parallel to the incident electric field, indicating dipole-type resonance, when the angle $\mathrm{O}$ is 0 degree. Similar phenomena were also observed at 10 degrees. For the second resonance of T-SRR01, there is electric and magnetic field coupling among units producing antiparallel currents parallel to the incident electric field. Therefore, the T-SRR01-2 with a fixed frequency of $1.8000 \mathrm{THz}$ is obtained when the angle $\mathrm{O}$ changes from 10 to 2 degrees. For the third resonance of T-SRR01, the charges are strongly concentrated at the ends of the arc-shaped " $T$ " cantilevers in the $x$-direction rather than being concentrated in the edge of " $\mathrm{T}$ " cantilevers in the $y$-direction. The magnetic fields are strongly concentrated on the edges of the arc-shaped " $T$ " cantilevers in the $x$-direction and the cantilever in the direction of the incident electric field, inducing parallel currents.

A similar phenomenon was also observed for the T-SRR02 structure. However, for the second resonance of T-SRR02, T-SRR02-2 has a stronger electric field-to-magnetic field coupling among units than T-SRR01-2, producing strongly parallel currents, which result in the larger tuning range of T-SRR02-2. Because it includes weaker currents than T-SRR01-3, T-SRR02-3 has a smaller tunable range.

These plasmonic modes are excited because of the nonnegligible spatial extension of the structure in comparison with the wavelength. The effective size of the structure increases as the cantilever bends down toward the substrate. Hence, the resonance frequency decreases as the cantilever bends downward. ${ }^{(25)}$ All the resonance ranges for T-SRR01 and T-RR02 resonator arrays have a red shift.

\section{Conclusions}

In summary, multiband MEMS-based metamaterial structures with polarizationindependent transmission behavior have been designed. When the angle $\mathrm{O}$ between the silicon and the cantilever is varied, two types of T-SRR exhibit tunable multiband transmission characteristics in the range from 0.5000 to $2.5000 \mathrm{THz}$. All the resonances for the T-SRR arrays have a redshift when the deformation angle $\mathrm{O}$ of the 10 to 0 cantilevers under thermal actuation decreases. Moreover, the resonant frequencies of T-SRR02 resonator arrays have a slight redshift compared with those of T-SRR01 resonator arrays. In the electric and magnetic field strength distributions at the resonance frequencies, the edge shape of the " $T$ " cantilevers plays an important role in transmission characteristics. The observed tunable multiband and polarization-independent properties may be desirable for potential applications in the development of $\mathrm{THz}$ devices. Furthermore, the approach presented here is not limited to $\mathrm{THz}$ frequencies but can be readily extended over the entire electromagnetic spectrum. 


\section{Acknowledgements}

This work was supported in part by the National Natural Science Foundation of China under Grant Nos. 61204119, 51035005, 61076107, 863 Program (2015AA043503), 973 Program (2013CB329401), the Shanghai Pujiang Talent Plan Sponsorship (No. 13PJ1405100), Innovation Program of Shanghai Municipal Education Commission under Grant No. 14ZZ019, and Department of Mechanical Electrical Engineering, Nanchang Institute of Technology, Nanchang 330099, China.

\section{References}

1 T. Ergin, N. Stenger, P. Brenner, J. B. Pendry and M. Wegener: Science 328 (2010) 337.

2 N. Fang and X. Zhang: Appl. Phys. Lett. 82 (2003) 161.

3 F. Dincer, M. Karaaslan, E. Unal and C. Sabah: Prog. Electromagn. Res. 141 (2013) 219.

4 W. Withayachumnankul, K. Jaruwongrungsee, A. Tuantranont, C. Fumeaux and D. Abbott: Sens. Actuators, A 189 (2013) 233.

5 N. I. Zheludev: Science 328 (2010) 582.

6 U. Leonhardt: Science 312 (2006) 1777.

7 T. Kaelberer, V. A. Fedotov, N. Papasimakis, D. P. Tsai and N. I. Zheludev: Science 330 (2010) 1510.

8 J. B. Pendry: Phys. Rev. Lett. 85 (2000) 3966.

9 N. Fang, H. Lee, C. Sun and X. Zhang: Science 308 (2005) 534.

10 D. Schurig, J. J. Mock, B. J. Justice, S. A. Cummer, J. B. Pendry, A. F. Starr and D. R. Smith: Science 314 (2006) 977.

11 E. Plum, J. Zhou, J. Dong, V. A. Fedotov, T. Koschny, C. M. Soukoulis and N. I. Zheludev: Phys. Rev. B 79 (2009) 035407.

12 X. Q. Zhang, Z. Tian, W. S. Yue, J. Q. Gu, S. Zhang, J. G. Han and W. L. Zhang: Adv. Mater. 25 (2013) 4567.

13 H.-T. Chen, J. F. O’Hara, A. K. Azad, A. J. Taylor and R. D. Averitt: Opt. Express 16 (2008) 7641.

14 J. N. Li, C. M. Shah, W. Withayachumnankul, B. S.-Y. Ung, A. Mitchell, S. Sriram, M. Bhaskaran, S. J. Chang and D. Abbott: Appl. Phys. Lett. 102 (2013) 121101.

15 Z. Han, H. Fujita and H. Toshiyoshi: Proc. 2012 Int. Conf. Opt. MEMS . Nanophotonics (OMN) (Banff, Canada, 6-9 August, 2012) pp. 27-28.

16 B. Ozbey and O. Aktas: Opt. Express 19 (2011) 5741.

17 T. Driscoll, S. Palit, M. M. Qazilbash, M. Brehm, F. Keilmann, B. Chae, S. J. Yun, H.-T. Kim, S. Y. Cho, N. M. Jokerst, D. R. Smith and D. N. Basov: Appl. Phys. Lett. 93 (2008) 024101.

18 Y. C. Jun and I. Brener: J. Opt. 14 (2012) 114013.

19 W. J. Padilla, A. J. Taylor, C. Highstrete, M. Lee and R. D. Averitt: Phys. Rev. Lett. 96 (2006) 107401.

20 H. T. Chen, H. Lu, A. K. Azad, R. D. Averitt, A. C. Gossard, S. A. Trugman, J. F. O'Hara and A. J. Taylor: Opt. Express 16 (2008) 7641.

21 Y. S. Lin, F. Ma and C. Lee: Opt. Lett. 38 (2013) 3126.

22 J. Han, A. Lakhtakia and C. W. Qiu. Terahertz: Opt. Express 16 (2008) 14390.

23 H. Tao, A. C. Strikwerda, K. Fan, W. J. Padilla, X. Zhang and R. D. Averitt: Phys. Rev. Lett. 103 (2009) 147401.

24 N. R. Han, Z. C. Chen, C. S. Lim, B. Ng and M. H. Hong: Opt. Express 19 (2011) 6990.

25 F. S. Ma, Y. S. Lin, X. H. Zhang and C. Lee: Sci. Appl. 3 (2014) 5. 